\title{
Some optimal entanglement-assisted quantum codes constructed from quaternary Hermitian linear complementary dual codes
}

\author{
Masaaki Harada*
}

\begin{abstract}
We establish the existence of optimal entanglement-assisted quantum $[[n, k, d ; n-k]]_{2}$ codes for $(n, k, d)=(14,6,7),(15,7,7),(17,6,9)$, $(17,7,8),(19,7,9)$ and $(20,7,10)$. These codes are obtained from quaternary Hermitian linear complementary dual codes. We also give some observation on the largest minimum weights.
\end{abstract}

\section{Introduction}

Let $\mathbb{F}_{q}$ denote the finite field of order $q$, where $q$ is a prime power. In this note, an $[n, k]_{q}$ code means a code over $\mathbb{F}_{q}$ of length $n$ and dimension $k$. The Euclidean dual code $C^{\perp}$ of an $[n, k]_{q}$ code $C$ is defined as $C^{\perp}=\left\{x \in \mathbb{F}_{q}^{n} \mid\right.$ $\langle x, y\rangle=0$ for all $y \in C\}$, where $\langle x, y\rangle=\sum_{i=1}^{n} x_{i} y_{i}$ for $x=\left(x_{1}, \ldots, x_{n}\right), y=$ $\left(y_{1}, \ldots, y_{n}\right) \in \mathbb{F}_{q}^{n}$. For any $x \in \mathbb{F}_{q^{2}}$, the conjugation of $x$ is defined as $\bar{x}=x^{q}$. The Hermitian dual code $C^{\perp_{H}}$ of an $[n, k]_{q^{2}}$ code $C$ is defined as $C^{\perp_{H}}=$ $\left\{x \in \mathbb{F}_{q^{2}}^{n} \mid\langle x, y\rangle_{H}=0\right.$ for all $\left.y \in C\right\}$, where $\langle x, y\rangle_{H}=\sum_{i=1}^{n} x_{i} \overline{y_{i}}$ for $x=$ $\left(x_{1}, \ldots, x_{n}\right), y=\left(y_{1}, \ldots, y_{n}\right) \in \mathbb{F}_{q^{2}}^{n}$. Let $\mathbf{0}_{n}$ denote the zero vector of length $n$. A code $C$ over $\mathbb{F}_{q}$ is called Euclidean linear complementary dual if $C \cap C^{\perp}=$ $\left\{\mathbf{0}_{n}\right\}$. A code $C$ over $\mathbb{F}_{q^{2}}$ is called Hermitian linear complementary dual if $C \cap C^{\perp_{H}}=\left\{\mathbf{0}_{n}\right\}$. These two families of codes are collectively called linear complementary dual (LCD for short) codes.

*Research Center for Pure and Applied Mathematics, Graduate School of Information Sciences, Tohoku University, Sendai 980-8579, Japan. email: mharada@tohoku.ac.jp. 
LCD codes were introduced by Massey [10] and gave an optimum linear coding solution for the two user binary adder channel. Recently, much work has been done concerning LCD codes for both theoretical and practical reasons. In particular, Carlet, Mesnager, Tang, Qi and Pellikaan [3] showed that any code over $\mathbb{F}_{q}$ is equivalent to some Euclidean LCD code for $q \geq 4$ and any code over $\mathbb{F}_{q^{2}}$ is equivalent to some Hermitian LCD code for $q \geq 3$. This motivates us to study Euclidean LCD codes over $\mathbb{F}_{q}(q=2,3)$ and quaternary Hermitian LCD codes. Here, we consider only the latter. In addition, it is known that quaternary Hermitian LCD codes give entanglement-assisted quantum $[[n, k, d ; n-k]]_{2}$ codes (see e.g. [4], [5], [7], [8] and [9] for background material on entanglement-assisted quantum codes). More precisely, if there is a Hermitian LCD $[n, k, d]_{4}$ code, then there is an entanglement-assisted quantum $[[n, k, d ; n-k]]_{2}$ code (see e.g. [7], [8] and [9]). From this point of view, quaternary Hermitian LCD codes play an important role in the study of entanglement-assisted quantum $[[n, k, d ; n-k]]_{2}$ codes. Note that quaternary Hermitian LCD codes are also called zero radical codes (see e.g. [7], [8] and [9]).

A Hermitian LCD $[n, k, d]_{4}$ code is called optimal if there is no Hermitian LCD $\left[n, k, d^{\prime}\right]_{4}$ code for $d^{\prime}>d$. An entanglement-assisted quantum $[[n, k, d ; c]]_{2}$ code is called optimal if there is no entanglement-assisted quantum $\left[\left[n, k, d^{\prime} ; c\right]\right]_{2}$ code for $d^{\prime}>d$. We denote the largest minimum weight $d$ by $d_{Q}(n, k)$. For $k \leq n \leq 20$, the current state of knowledge about $d_{Q}(n, k)$ are listed in [5, Table II] and [9, Table 6]. Many optimal entanglementassisted quantum $[[n, k, d ; n-k]]_{2}$ codes codes are constructed from optimal quaternary Hermitian LCD codes. As a contribution in this direction, in this note, we establish the existence of optimal entanglement-assisted quantum $[[n, k, d ; n-k]]_{2}$ codes for

$$
(n, k, d)=(14,6,7),(15,7,7),(17,6,9),(17,7,8),(19,7,9),(20,7,10) .
$$

From [5, Table II] and [9, Table 6], we determine the largest minimum weight as follows:

$$
\begin{aligned}
& d_{Q}(14,6)=d_{Q}(15,7)=7, d_{Q}(17,6)=d_{Q}(19,7)=9, \\
& d_{Q}(17,7)=8 \text { and } d_{Q}(20,7)=10 .
\end{aligned}
$$

In addition, we establish the existence of an entanglement-assisted quantum $[[20,8,9 ; 12]]_{2}$ code. We also give some observation on the largest minimum weights for Hermitian LCD $[n, k]_{4}$ codes for $k=n-1, n-2$ and $n-3$.

All computer calculations in this note were done by Magma [1]. 


\section{New optimal codes}

\subsection{Optimal quaternary Hermitian LCD codes}

We denote the finite field of order 4 by $\mathbb{F}_{4}=\left\{0,1, \omega, \omega^{2}\right\}$, where $\omega^{2}=\omega+1$. A linear $[n, k]_{4}$ code $C$ is a $k$-dimensional vector subspace of $\mathbb{F}_{4}^{n}$. All codes over $\mathbb{F}_{4}$ in this note are linear. A code over $\mathbb{F}_{4}$ is called quaternary. The weight $\operatorname{wt}(x)$ of a vector $x \in \mathbb{F}_{4}^{n}$ is the number of non-zero components of $x$. A vector of $C$ is called a codeword of $C$. The minimum non-zero weight of all codewords in $C$ is called the minimum weight $d(C)$ of $C$. An $[n, k, d]_{4}$ code is an $[n, k]_{4}$ code with minimum weight $d$. Two $[n, k]_{4}$ codes $C$ and $C^{\prime}$ are equivalent if there is an $n \times n$ monomial matrix $P$ over $\mathbb{F}_{4}$ with $C^{\prime}=\{x P \mid x \in C\}$.

Every $[n, k, d]_{4}$ code is equivalent to a code with generator matrix of the form $\left(\begin{array}{ll}I_{k} & A\end{array}\right)$, where $A$ is a $k \times(n-k)$ matrix and $I_{k}$ denotes the identity matrix of order $k$. Let $r_{i}$ be the $i$-th row of $A$. Here, we may assume that $A$ satisfies the following conditions:

(i) $r_{1}=\left(\mathbf{0}_{n-k-d+1}, \mathbf{1}_{d-1}\right)$, where $\mathbf{1}_{s}$ denotes the all-one vector of length $s$,

(ii) $\operatorname{wt}\left(r_{i}\right) \geq d-1$,

(iii) the first nonzero element of $r_{i}$ is 1 ,

(iv) $r_{1}<r_{2}<\cdots<r_{k}$ if $d \geq 3$ and $r_{1} \leq r_{2} \leq \cdots \leq r_{k}$ if $d \leq 2$,

where we consider some order $<$ on the set of vectors of length $n-k$. The set of matrices $A$ is constructed, row by row, under the condition that the minimum weight of the $[n+m-k, m]_{4}$ code with generator matrix

$$
\left(\begin{array}{cc} 
& r_{1} \\
I_{m} & \vdots \\
& r_{m}
\end{array}\right)
$$

is at least $d$ for each $m=2,3, \ldots, k-1$. It is obvious that the set of all $[n, k, d]_{4}$ codes obtained in this approach contains a set of all inequivalent $[n, k, d]_{4}$ codes. It is known that a quaternary code $C$ is Hermitian LCD if and only if $G \bar{G}^{T}$ is nonsingular for a generator matrix $G$ of $C$, where $A^{T}$ and $\bar{A}$ denote the transposed matrix and the conjugate matrix for a matrix $A$, respectively. In addition, it is known that a quaternary code $C$ is Hermitian LCD if and only if $C^{\perp_{H}}$ is Hermitian LCD (see e.g. [3] and [7]). 
By the above approach, our exhaustive computer search found a Hermitian LCD code with parameters $[15,7,7]_{4},[17,6,9]_{4},[17,7,8]_{4}$ and $[20,7,10]_{4}$. We denote these codes by $C_{15}, C_{17,1}, C_{17,2}$ and $C_{20}$, respectively. These codes have generator matrices $\left(\begin{array}{ll}I_{7} & M_{15}\end{array}\right),\left(\begin{array}{ll}I_{6} & M_{17,1}\end{array}\right),\left(\begin{array}{ll}I_{7} & M_{17,2}\end{array}\right)$ and $\left(\begin{array}{ll}I_{7} & M_{20}\end{array}\right)$, respectively, where $M_{15}, M_{17,1}, M_{17,2}$ and $M_{20}$ are listed in Figure 1 .

Let $C$ be an $[n, k]_{4}$ code. A shortened code of $C$ on the coordinate $i$ is the set of all codewords in $C$ which are 0 in the $i$-th coordinate with that coordinate deleted. We denote the code by $S(C, i)$. A punctured code of $C$ on the coordinate $i$ is the code obtained from $C$ by deleting the $i$-th coordinate. Let $C_{14}$ be the code $S\left(C_{15}, 4\right)$. We verified that $C_{14}$ is a Hermitian LCD $[14,6,7]_{4}$ code. We denote by $C_{19}$ the punctured code of $C_{20}$ on the first coordinate. We verified that $C_{19}$ is a Hermitian LCD $[19,7,9]_{4}$ code.

Therefore, we have the following result.

Proposition 1. There is a Hermitian $L C D[n, k, d]_{4}$ code for

$$
(n, k, d)=(14,6,7),(15,7,7),(17,6,9),(17,7,8),(19,7,9),(20,7,10) .
$$

The weight enumerator of an $[n, k]_{4}$ code $C$ is defined as $\sum_{i=0}^{n} A_{i} y^{i}$, where $A_{i}$ denotes the number of codewords of weight $i$ in $C$. The weight enumerators of the codes $C_{14}, C_{15}, C_{17,1}, C_{17,2}, C_{19}$ and $C_{20}$ are listed in Table 1.

\subsection{Optimal entanglement-assisted quantum codes}

An entanglement-assisted quantum $[[n, k, d ; c]]_{2}$ code $\mathcal{C}$ encodes $k$ information qubits into $n$ channel qubits with the help of $c$ pairs of maximally entangled Bell states. The parameter $d$ is called the minimum weight of $\mathcal{C}$. The entanglement-assisted quantum code $\mathcal{C}$ can correct up to $\left\lfloor\frac{d-1}{2}\right\rfloor$ errors acting on the $n$ channel qubits (see e.g. [7] and [9]). An entanglement-assisted quantum $[[n, k, d ; 0]]_{2}$ code is a standard quantum code. If there is a Hermitian LCD $[n, k, d]_{4}$ code, then there is an entanglement-assisted quantum $[[n, k, d ; n-k]]_{2}$ code (see e.g. [7] and [9]).

An entanglement-assisted quantum $[[n, k, d ; c]]_{2}$ code is called optimal if there is no entanglement-assisted quantum $\left[\left[n, k, d^{\prime} ; c\right]\right]_{2}$ code for $d^{\prime}>d$. We denote the largest minimum weight $d$ by $d_{Q}(n, k)$. The largest minimum weights $d_{Q}(n, k)$ have been widely studied in [5] for $n \leq 20$. The current state of knowledge about $d_{Q}(n, k)$ can be found in [5, Table II] and [9, Table 6] for 


$$
\begin{aligned}
& M_{15}=\left(\begin{array}{cccccccc}
0 & 0 & 1 & 1 & 1 & 1 & 1 & 1 \\
1 & 0 & 1 & \omega^{2} & \omega^{2} & 1 & 0 & \omega \\
1 & 1 & 0 & 1 & \omega & 1 & 1 & 0 \\
1 & 1 & \omega & 1 & \omega^{2} & 0 & \omega & \omega^{2} \\
1 & \omega & 0 & 1 & 0 & \omega & \omega^{2} & \omega^{2} \\
1 & \omega & \omega^{2} & \omega^{2} & 1 & \omega & 1 & 0 \\
1 & \omega^{2} & \omega^{2} & 0 & 0 & \omega & \omega & \omega
\end{array}\right) \\
& M_{17,1}=\left(\begin{array}{ccccccccccc}
0 & 0 & 0 & 1 & 1 & 1 & 1 & 1 & 1 & 1 & 1 \\
1 & 1 & 1 & \omega & 1 & 1 & 1 & 1 & 0 & 0 & 0 \\
1 & \omega & \omega & 1 & \omega & 1 & 0 & 0 & 1 & 1 & 0 \\
1 & \omega & \omega^{2} & \omega & 1 & 0 & \omega & 0 & 1 & 0 & 1 \\
1 & \omega^{2} & 0 & 1 & 0 & \omega & \omega^{2} & 1 & 1 & \omega & \omega \\
1 & \omega^{2} & \omega & \omega & 0 & 0 & \omega & 1 & \omega & 1 & 0
\end{array}\right) \\
& M_{17,2}=\left(\begin{array}{cccccccccc}
0 & 0 & 0 & 1 & 1 & 1 & 1 & 1 & 1 & 1 \\
0 & 1 & \omega^{2} & \omega & 1 & 0 & \omega^{2} & \omega^{2} & \omega & 1 \\
0 & 1 & \omega & 0 & 1 & 1 & 0 & 1 & \omega^{2} & \omega^{2} \\
1 & 0 & \omega & \omega & 0 & \omega & 1 & \omega & 1 & 0 \\
1 & 0 & \omega^{2} & 0 & 1 & 1 & 0 & \omega & \omega & \omega \\
1 & 1 & 1 & 0 & \omega & \omega^{2} & \omega & 0 & 1 & \omega^{2} \\
1 & 1 & \omega & \omega^{2} & \omega & \omega & 1 & 0 & \omega & 1
\end{array}\right) \\
& M_{20}=\left(\begin{array}{ccccccccccccc}
0 & 0 & 0 & 0 & 1 & 1 & 1 & 1 & 1 & 1 & 1 & 1 & 1 \\
1 & 0 & \omega^{2} & \omega & 1 & \omega & 0 & \omega & 1 & \omega & 1 & 0 & 0 \\
1 & 1 & 0 & 1 & 0 & 0 & \omega & \omega^{2} & \omega & \omega & 1 & \omega & 1 \\
1 & 1 & \omega & \omega & \omega & 1 & 1 & 0 & 0 & 1 & 1 & 0 & 0 \\
1 & 1 & \omega^{2} & 1 & \omega & \omega & \omega & \omega^{2} & 1 & 0 & \omega & 1 & 0 \\
1 & \omega & 1 & 1 & 1 & 1 & 1 & 1 & 1 & 0 & 0 & 0 & 0 \\
1 & \omega^{2} & \omega & \omega^{2} & 1 & \omega & 0 & 1 & 0 & 1 & 0 & 1 & 0
\end{array}\right)
\end{aligned}
$$

Figure 1: Matrices $M_{15}, M_{17,1}, M_{17,2}$ and $M_{20}$ 
Table 1: Weight enumerators

\begin{tabular}{|c|c|}
\hline Code & Weight enumerator \\
\hline$C_{14}$ & $\begin{array}{l}1+210 y^{7}+252 y^{8}+588 y^{9}+945 y^{10}+882 y^{11}+819 y^{12} \\
+336 y^{13}+63 y^{14}\end{array}$ \\
\hline$C_{15}$ & $\begin{array}{l}1+336 y^{7}+756 y^{8}+1323 y^{9}+2415 y^{10}+4095 y^{11}+3759 y^{12} \\
+2289 y^{13}+1197 y^{14}+213 y^{15}\end{array}$ \\
\hline$C_{17,1}$ & $\begin{array}{l}1+201 y^{9}+279 y^{10}+492 y^{11}+777 y^{12}+840 y^{13}+849 y^{14} \\
+456 y^{15}+174 y^{16}+27 y^{17}\end{array}$ \\
\hline$C_{17,2}$ & $\begin{array}{l}1+204 y^{8}+549 y^{9}+1053 y^{10}+1977 y^{11}+3117 y^{12}+3711 y^{13} \\
+3111 y^{14}+1875 y^{15}+642 y^{16}+144 y^{17}\end{array}$ \\
\hline$C_{19}$ & $\begin{array}{l}1+111 y^{9}+423 y^{10}+801 y^{11}+1509 y^{12}+2595 y^{13}+3291 y^{14} \\
+3315 y^{15}+2502 y^{16}+1362 y^{17}+402 y^{18}+72 y^{19}\end{array}$ \\
\hline$C_{20}$ & $\begin{array}{l}1+297 y^{10}+441 y^{11}+978 y^{12}+1767 y^{13}+2685 y^{14}+3381 y^{15} \\
+3078 y^{16}+2349 y^{17}+1038 y^{18}+318 y^{19}+51 y^{20}\end{array}$ \\
\hline
\end{tabular}

$n \leq 20$. From [5, Table II] and [9, Table 6], we have the following:

$$
\begin{aligned}
& d_{Q}(14,6)=6 \text { or } 7, \quad d_{Q}(15,7)=6 \text { or } 7, \\
& d_{Q}(17,6)=8 \text { or } 9, \quad d_{Q}(17,7)=7 \text { or } 8, \\
& d_{Q}(19,7)=8 \text { or } 9, \quad d_{Q}(20,7)=9 \text { or } 10 .
\end{aligned}
$$

Therefore, from quaternary Hermitian LCD codes given in Proposition 1, we have the following:

Proposition 2. (i) There is an optimal entanglement-assisted quantum $[[n, k, d ; n-k]]_{2}$ code from a Hermitian $L C D[n, k, d]_{4}$ code for

$$
(n, k, d)=(14,6,7),(15,7,7),(17,6,9),(17,7,8),(19,7,9),(20,7,10) .
$$

$$
\begin{aligned}
& d_{Q}(14,6)=d_{Q}(15,7)=7, \quad d_{Q}(17,6)=d_{Q}(19,7)=9, \\
& d_{Q}(17,7)=8, \quad d_{Q}(20,7)=10 .
\end{aligned}
$$

Let $d_{4}(n, k)$ denote the largest minimum weight among all Hermitian LCD $[n, k]_{4}$ codes. From [5, Table II] and [9, Table 6], it is known that $d_{4}(14,6) \leq$ $7, d_{4}(15,7) \leq 7, d_{4}(17,6) \leq 9, d_{4}(17,7) \leq 8, d_{4}(19,7) \leq 9$ and $d_{4}(20,7) \leq 10$. Hence, quaternary Hermitian LCD codes listed in Proposition 1 are optimal.

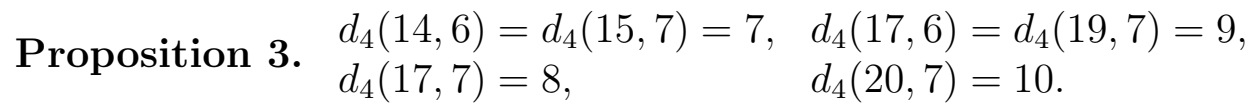




\subsection{Largest minimum weights}

From [5, Table II] and [9, Table 6], it is known that $d_{Q}(12,6)=5$ or 6 . By the approach given in the beginning of this section, our exhaustive search shows that there is no Hermitian LCD $[12,6,6]_{4}$ code. In addition, our exhaustive computer search found a Hermitian LCD $[12,6,5]_{4}$ code $D_{12}$. The code $D_{12}$ has generator matrix $\left(\begin{array}{ll}I_{6} & N_{12}\end{array}\right)$, where

$$
N_{12}=\left(\begin{array}{cccccc}
0 & 0 & 1 & 1 & 1 & 1 \\
0 & 1 & 0 & \omega^{2} & \omega & \omega^{2} \\
1 & 1 & 0 & \omega & 0 & 1 \\
1 & 1 & \omega^{2} & \omega & \omega^{2} & \omega^{2} \\
1 & \omega & 0 & \omega & \omega & \omega \\
1 & \omega^{2} & 0 & 1 & 1 & 0
\end{array}\right)
$$

The weight enumerator of $D_{12}$ is given by:

$$
1+72 y^{5}+177 y^{6}+378 y^{7}+792 y^{8}+1044 y^{9}+999 y^{10}+522 y^{11}+111 y^{12} .
$$

Proposition 4. $d_{4}(12,6)=5$.

It is worthwhile to determine whether there is a entanglement-assisted quantum $[[12,6,6 ; 6]]_{2}$ code.

From [5, Table II] and [9, Table 6], it is known that $d_{Q}(20,8)=8,9$ or 10 . By the approach given in the beginning of this section, our computer search found a Hermitian LCD $[20,8,9]_{4}$ code $D_{20}$. The code $D_{20}$ has generator matrix $\left(\begin{array}{cc}I_{8} & N_{20}\end{array}\right)$, where

$$
N_{20}=\left(\begin{array}{cccccccccccc}
0 & 0 & 0 & 0 & 1 & 1 & 1 & 1 & 1 & 1 & 1 & 1 \\
1 & 1 & 1 & \omega & 1 & 1 & 0 & 0 & 1 & 1 & 0 & 0 \\
1 & 1 & \omega & 1 & \omega & 0 & 1 & 0 & 1 & 0 & 1 & 0 \\
1 & \omega & \omega^{2} & 1 & 1 & 1 & 1 & 1 & 0 & 0 & 0 & 0 \\
1 & \omega & 1 & 0 & \omega & 0 & \omega & 1 & \omega & 1 & 0 & 0 \\
1 & \omega^{2} & \omega^{2} & \omega & 0 & \omega & 0 & 1 & \omega & 0 & 1 & 0 \\
1 & \omega^{2} & \omega & 0 & 1 & \omega^{2} & 0 & 1 & 1 & 0 & 0 & 1 \\
1 & \omega^{2} & 1 & \omega^{2} & 1 & 0 & \omega & \omega & 0 & \omega & 1 & 1
\end{array}\right)
$$

The weight enumerator of $D_{20}$ is given by:

$$
\begin{aligned}
1+288 y^{9}+714 y^{10}+1725 y^{11}+3888 y^{12}+7272 y^{13}+11208 y^{14} \\
+13338 y^{15}+12423 y^{16}+8640 y^{17}+4446 y^{18}+1377 y^{19}+216 y^{20}
\end{aligned}
$$


Proposition 5. (i) There is a Hermitian LCD $[20,8,9]_{4}$ code and there is an entanglement-assisted quantum $[[20,8,9 ; 12]]_{2}$ code.

(ii) $d_{4}(20,8)=9$ or 10 and $d_{Q}(20,8)=9$ or 10 .

$3 \quad d_{4}(n, k)$ for $k=n-1, n-2, n-3$

In this section, we study $d_{4}(n, k)$ for $k=n-1, n-2, n-3$.

Let $C$ be an $[n, n-1]_{4}$ code. We may assume without loss of generality that $C$ has generator matrix of the following form:

$$
\left(\begin{array}{cc} 
& a_{1} \\
I_{n-1} & \vdots \\
& a_{n-1}
\end{array}\right)
$$

where $a_{i} \in\{0,1\}(i=1,2, \ldots, n-1)$ and $a=\left(a_{1}, a_{2}, \ldots, a_{n-1}\right)$. The matrix $\left(\overline{a_{1}} \cdots \overline{a_{n-1}} 1\right)$ is a generator matrix of $C^{\perp_{H}}$. It follows that $C^{\perp_{H}}$ is Hermitian LCD if and only if $\operatorname{wt}(a) \equiv 0(\bmod 2)$. In other words, $C$ is Hermitian LCD if and only if $\operatorname{wt}(a) \equiv 0(\bmod 2)$. Hence, we have the following:

Proposition 6. Suppose that $n \geq 2$. Then

$$
d_{4}(n, n-1)= \begin{cases}1 & \text { if } n \text { is even }, \\ 2 & \text { if } n \text { is odd. }\end{cases}
$$

The following lemma is a key idea for the determination of $d_{4}(n, n-2)$ and $d_{4}(n, n-3)$.

Lemma 7. Let $i$ be an integer with $2 \leq i<n$. If $n>\frac{4^{i}-1}{3}$, then $d_{4}(n, n-i)=$ 2 .

Proof. Let $C$ be an $[n, n-i, d]_{4}$ code with generator matrix of the form:

$$
G=\left(\begin{array}{cccccc} 
& 1 & 1 & 0 & \cdots & 0 \\
& \vdots & \vdots & \vdots & & \vdots \\
I_{n-i} & 1 & 1 & 0 & \cdots & 0
\end{array}\right)
$$

Since $G \bar{G}^{T}=I_{n-i}, C$ is Hermitian LCD. By the construction, it is trivial that $C$ has minimum weight 2. By the sphere-packing bound, if $d \geq 3$, then $n \leq \frac{4^{i}-1}{3}$. The result follows. 


\section{Proposition 8.}

$$
d_{4}(n, n-2)= \begin{cases}3 & \text { if } n=3 \\ 2 & \text { if } n \geq 4\end{cases}
$$

Proof. It is known that $d_{4}(3,1)=3, d_{4}(4,2)=2$ and $d_{4}(5,3)=2$ [9]. If $n \geq 6$, then $d_{4}(n, n-2)=2$ by Lemma 7 .

\section{Proposition 9.}

$$
d_{4}(n, n-3)= \begin{cases}3 & \text { if } 4 \leq n \leq 18 \\ 2 & \text { if } n \geq 19\end{cases}
$$

Proof. It is known that $d_{4}(n, n-3)=3$ for $n=4,5, \ldots, 8$ [9]. If $n \geq 22$, then $d_{4}(n, n-3)=2$ by Lemma 7 .

It is known that the largest minimum weight among (unrestricted) $[n, n-$ $3]_{4}$ codes is 3 for $n=9,10, \ldots, 21$. By the approach given in the beginning of the previous section, our exhaustive search shows that there is no Hermitian LCD $[n, n-3,3]_{4}$ code for $n=19,20,21$. Let $E_{n}(n=19,20,21)$ be the $[n, n-3]_{4}$ code with generator matrix of the form:

$$
\left(\begin{array}{cccc} 
& 1 & 1 & 0 \\
I_{n-3} & \vdots & \vdots & \vdots \\
& 1 & 1 & 0
\end{array}\right)
$$

As described in the proof of Lemma 7, $E_{n}(n=19,20,21)$ is a Hermitian LCD $[n, n-3,2]_{4}$ code.

Let $E_{18}$ be the $[18,15]_{4}$ code with generator matrix $\left(\begin{array}{ll}I_{15} & L_{18}\end{array}\right)$, where

$$
L_{18}^{T}=\left(\begin{array}{ccccccccccccccc}
0 & 0 & 0 & 1 & 1 & 1 & 1 & 1 & 1 & 1 & 1 & 1 & 1 & 1 & 1 \\
1 & 1 & 1 & 0 & 0 & 0 & 1 & 1 & 1 & \omega & \omega & \omega & \omega & \omega^{2} & \omega^{2} \\
1 & \omega & \omega^{2} & 1 & \omega & \omega^{2} & 0 & 1 & \omega^{2} & 0 & 1 & \omega & \omega^{2} & \omega & \omega^{2}
\end{array}\right) .
$$

We define the codes $E_{i}(i=17,16, \ldots, 9)$ by the shortened codes as follows:

$$
\begin{aligned}
S\left(E_{18}, 1\right), S\left(E_{17}, 2\right), S\left(E_{16}, 1\right), S\left(E_{15}, 4\right), S\left(E_{14}, 1\right), & \\
& S\left(E_{13}, 2\right), S\left(E_{12}, 1\right), S\left(E_{11}, 2\right), S\left(E_{10}, 2\right),
\end{aligned}
$$

respectively. We verified that $E_{n}$ is a Hermitian $\operatorname{LCD}[n, n-3,3]_{4}$ code for $n=9,10, \ldots, 18$. The result follows. 
Acknowledgment. This work was supported by JSPS KAKENHI Grant Numbers $15 \mathrm{H} 03633$ and 19H01802. The author would like to thank the anonymous referee and the editor Markus Grassl for the useful comments.

\section{References}

[1] W. Bosma, J. Cannon and C. Playoust, The Magma algebra system I: The user language, J. Symbolic Comput. 24 (1997), 235-265.

[2] T.A. Brun, I. Devetak and M.H. Hsieh, Correcting quantum errors with entanglement, Science $\mathbf{3 1 4}$ (2006), 436-439.

[3] C. Carlet, S. Mesnager, C. Tang, Y. Qi and R. Pellikaan, Linear codes over $\mathbb{F}_{q}$ are equivalent to LCD codes for $q>3$, IEEE Trans. Inform. Theory 64 (2018), 3010-3017.

[4] X. Chen, S. Zhu and X. Kai, Entanglement-assisted quantum MDS codes constructed from constacyclic codes, Quantum Inf. Process. 17 (2018), Art. 273, 18 pp.

[5] C.-Y. Lai and A. Ashikhmin, Linear programming bounds for entanglement-assisted quantum error-correcting codes by split weight enumerators, IEEE Trans. Inform. Theory 64 (2018), 622-639.

[6] C.-Y. Lai, T.A. Brun and M.M. Wilde, Dualities and identities for entanglement-assisted quantum codes, Quantum Inf. Process. 13 (2014), 957-990.

[7] R. Li, X. Li and L. Guo, On entanglement-assisted quantum codes achieving the entanglement-assisted Griesmer bound, Quantum Inf. Process. 14 (2015), 4427-4447.

[8] L. Lu, R. Li and L. Guo, Entanglement-assisted quantum codes from quaternary codes of dimension five, Int. J. Quantum Inf. 15 (2017), 1750017,28 pp.

[9] L. Lu, R. Li, L. Guo and Q. Fu, Maximal entanglement entanglementassisted quantum codes constructed from linear codes, Quantum Inf. Process. 14 (2015), 165-182. 
[10] J.L. Massey, Linear codes with complementary duals, Discrete Math. 106/107 (1992), 337-342. 